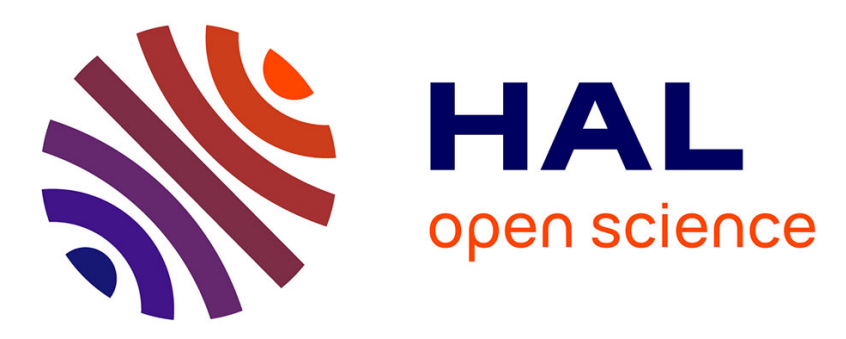

\title{
Répétitions des discours sur la culture scientifique et technique et effets de la catégorisation scientifique scolaire
}

\author{
Olivier Las Vergnas
}

\section{- To cite this version:}

Olivier Las Vergnas. Répétitions des discours sur la culture scientifique et technique et effets de la catégorisation scientifique scolaire. Innovations - Revue d'économie et de management de l'innovation, 2017, Techno-sciences en société, 2017/1 (52), pp 85-109. 10.3917/inno.052.0085 • hal-01469360

\author{
HAL Id: hal-01469360 \\ https://hal.science/hal-01469360
}

Submitted on 16 May 2018

HAL is a multi-disciplinary open access archive for the deposit and dissemination of scientific research documents, whether they are published or not. The documents may come from teaching and research institutions in France or abroad, or from public or private research centers.
L'archive ouverte pluridisciplinaire HAL, est destinée au dépôt et à la diffusion de documents scientifiques de niveau recherche, publiés ou non, émanant des établissements d'enseignement et de recherche français ou étrangers, des laboratoires publics ou privés. 


\title{
Répétitions des discours sur la culture scientifique et technique et effets de la catégorisation scientifique scolaire
}

\author{
Olivier Las Vergnas ${ }^{1,2,3}$
}

\author{
${ }^{1}$ Professeur à l'Université de Lille (F59000, CIREL, EA 4354) \\ ${ }^{2}$ Directeur de recherches doctorales, invité à l'Université de Nanterre (CREF-AFA, EA 1589) \\ ${ }^{3}$ Président de l'Association française d'Astronomie \\ https://www.cairn.info/revue-innovations-2017-1-page-85.htm \\ Paru dans : Innovations, 52,(1), 85-109. doi:10.3917/inno.052.0085.
}

\section{Résumé :}

Les plus récents rapports ou programmes politiques concernant les cultures scientifiques, techniques et industrielles (CSTI) en France continuent de répéter les mêmes analyses et les préconisations que celles déjà formulées régulièrement dans ce type de discours depuis plusieurs décennies. Ils ne prennent pas plus en compte que leurs prédécesseurs le fait que le système d'enseignement secondaire français crée avec le Bac S une catégorisation déterminante pour tous les élèves, du point de vue de la reconnaissance comme «scientifiques » ou non. Sachant que ce diplôme clef réserve chaque année le titre de «scientifique » à un seul quart de chaque génération, le champ des actions de la CSTI pour adultes devrait alors s'analyser en séparant deux groupes aux objectifs divergents : l'un ne remettant pas en cause les effets a posteriori de cette catégorisation mais les confirmant, l'autre favorisant a contrario l'appropriation de savoirs et de méthodes qui transgressent ce clivage scolaire. Or il n'en est rien et le champ de la CSTI continue de n'être qu'un agglomérat d'actions aux objectifs sociaux disparates. Plus encore, la multiplication de jeux d'acteurs focalisés sur des objectifs et des enjeux très spécifiques -liés en particulier à l'idée de développer localement des réseaux d'innovations technologiques- rend plus que jamais difficile le repérage des effets socio-culturels néfastes en France résultant de cette trop forte sélection par le Bac S. De fait, ceux-ci perdurent et la catégorisation scientifique scolaire constitue plus que jamais, un invariant macrosocial inhibant le partage de l'esprit d'investigation et d'innovation technoscientifique.

Mots clefs: Culture scientifique, catégorisation scientifique scolaire, innovations technologiques, baccalauréat scientifique, jeux d'acteurs. 


\section{Abstract:}

In France, latest reports and political programs on public understanding of science (in French: culture scientifique, technique et industrielle, CSTI) continue to repeat the same analysis and recommendations than those already made regularly in this type of discourses for decades. They do not take into account more than their predecessors that the French secondary education system creates a students' categorization in terms of recognition as "scientific" with the "Bac S", a key diploma allowing each year only a quarter of each generation to be later considered as "scientists". CSTI for adults should therefore be separated in two families: the first organizing the dialogue between scientists and "laymen" without questioning this divide and the second fostering the appropriation of knowledge in order to transgress this scientific school divide. Now it is not so, the field of CSTI continuing to be only a patchwork of various actions with disparate social aims. Moreover, the multiplication of actors focused on very specific objectives -such as promoting local innovation networks- makes more than ever difficult to identify bad sociocultural effects at a nationwide scale. Due to this attitude, school scientific divide is more than ever a macro-invariant inhibiting the sharing of sprit of inquiry and technological innovation.

Cet article est soumis dans le cadre de l'appel à contribution :

Technosciences, économie, société : tensions entre «culture émancipatrice» et innovation triomphante

Innovations, 2017 Diffusion interdite.

https://www.cairn.info/revue-innovations-2017-1-page-85.htm

Pour le citer:

Las Vergnas, O. (2017). Répétitions des discours sur la culture scientifique et technique et effets de la catégorisation scientifique scolaire. Innovations, 52,(1), 85-109.

doi:10.3917/inno.052.0085. 


\section{Répétitions des discours sur la culture scientifique et technique et effets de la catégorisation scientifique scolaire}

Olivier Las Vergnas,

Professeur à l'Université de Lille - Sciences et Technologies

(équipe CIREL-Trigone, EA 4354),

\section{Introduction et méthodologie}

Cette contribution se fonde sur une analyse des dernières préconisations concernant les cultures scientifiques, techniques et industrielles en France (OPECST, 2014), conduite avec le prisme des constats préalablement établis d'une vaine répétition de ce type de discours depuis un demisiècle (Labasse, 1999, Schiele 2006) et de l'interprétation de cette répétition par des effets de catégorisation de la population scolaire en scientifiques et non scientifiques (Las Vergnas, $2011 \mathrm{a}$; 2011b). Ce travail confirme le fait que loin de tirer profit de ces constats antérieurs pour questionner cette catégorisation scolaire, les récents rapports ne font que continuer de reproduire des préconisations qui se sont systématiquement révélées inefficaces ; plus encore, il est constaté que la multiplication de jeux d'acteurs focalisés sur des objectifs et des enjeux disparates, rend plus que jamais difficile le repérage des effets socio-culturels néfastes en France d'une trop forte sélection par le Bac S.

\section{Rappel du contexte : la CSTI en butte à la catégorisation scolaire}

\subsection{La vaine répétition des discours de culture scientifique pour tous}

En France, comme dans beaucoup de pays dits développés, les discours politiques d'injonction à la «culture scientifique, technique et industrielle » (CSTI) pour tous n'ont cessé de se répéter depuis 40 ans. Pourtant, pendant cette même période, se sont multipliées les initiatives préconisées dans ces mêmes discours et on a assisté non seulement au développement d'actions ponctuelles mais aussi à la mise en place de réseaux nationaux de ressources, de centres permanents et d'instruments de politiques systématiques (Las Vergnas, 2011b ; Brand-Grau, Claerr \& Smith, 2015). Or, si ces discours se répètent, c'est que ces solutions se sont révélées inopérantes: force est de constater, année après année, qu'il n'a pas suffi de décréter officiellement prioritaire cette «CSTI pour tous» et de démultiplier les dispositifs de 
sensibilisation ou de médiation pour modifier de manière globalement significative les représentations sociales de la Science (Schiele, 2005).

Ce constat d'impuissance a lui-même été partagé par de nombreux acteurs et depuis 1999 c'est la dénonciation de la vaine répétition des discours de CSTI qui elle-même se répète ${ }^{1}$, dans une saisissante mise en abyme (Labasse, 1999; Schiele, 2005). Certes, entre temps, quelques éléments rhétoriques ont évolué, comme le modèle dit «du déficit », (au sens d'un manque de connaissances à combler) que plusieurs acteurs jugeaient trop scolairement pédagogique (Callon, 1998) et qui a laissé la place à une formulation plus participative s'appuyant sur l'idée de l'organisation du partage de la «science en société » (Felt, 2010) sans que cela n'empêche en rien les discours officiels d'injonction de continuer à se répéter. L'année 2014 n'a pas été en reste, avec la publication d'un copieux rapport de l'office parlementaire pour l'évaluation des choix scientifiques et techniques (OPECST, 2014) intitulé «les cultures scientifiques techniques et industrielles, un impératif », emblématique de cette filiation et présentant lui-même en première annexe une liste des rapports précédents (p 271).

Un des aspects clefs de tous ces discours est qu'ils articulent deux objets qui se révèlent aussi ambigus l'un que l'autre, à savoir la (ou les) «culture(s) scientifique et technique et industrielle » d'une part et une présumée «désaffection des sciences » (ou des filières scientifiques) $)^{2}$, d'autre part. Le propos n'est pas ici de revenir en détail sur ces ambigüités qui ont d'ores et déjà été analysées dans plusieurs publications (en particulier Schiele, 2005 ; Las Vergnas 2011a pour les discours sur la CSTI et Doray, Gemme \& Gibaud, 2003, Convert, 2006 ; Las Vergnas, 2006 et HCST \& CEREQ, 2015 pour ceux sur la désaffection).

Sur le fond, aussi bien termes de constats que de préconisations, ces discours n'évoluent guère d'une année sur l'autre : tous s'inquiètent donc d'une «désaffection » sans vraiment en stabiliser une ou des définitions ${ }^{3}$, trouvent les sciences scolaires trop abstraites, la TV inadaptée

\footnotetext{
${ }^{1}$ Ce rappel des travaux antérieurs reprend donc en partie certains constats déjà publiés (Las Vergnas, 2011a ; 2011b) qui ont été ici très largement actualisés. Ceux-ci se fondent sur une analyse des discours et non sur une évaluation des actions ou sur une critique de la pertinence ou non de tel ou tel discours, posture qui a été reprise pour le présent article.

${ }^{2}$ A noter que le passage au pluriel de «cultures » dans le titre du rapport de l'OPESCT (2014) s'inscrit dans une volonté d'encourager une appréhension différentielle des dimensions « scientifique, technique et industrielle », mais que celle-ci se révèle circonscrite à l'introduction (p15-16), d'autant que l'utilisation de « technique » plutôt que « technologique » n'est pas commentée,

${ }^{3}$ Suggérant par voie de conséquence le plus souvent un lien de causalité entre une baisse constatée des effectifs des
} 
à son présumé rôle éducatif et la fonction de vulgarisation des chercheurs insuffisamment reconnue (voir par exemple Blandin \& Renar, 2003). Certes, ces dernières années, la part consacrée à des discours sur la présumée « désaffection des filières scientifiques » a bien dû être questionnée puisque explicitement contredite par la pénurie de plus en plus criante de postes de chercheurs, sans pour autant disparaître des discours ${ }^{4}$. En parallèle, s'est accrue la place accordée à ce qui est qualifié de «nouvelle donne » résultant de la société de la connaissance numérique : bien que celle-ci a permis de développer de nouveaux outils de diffusion (Blogs, réseaux sociaux, Moocs, Serious Games, applications connectées) ou des nouveaux partenariats (forums, communautés virtuelles, FabLabs), elle reste regardée comme étant finalement « d'une portée contrastée »(OPECST, 2014), en particulier en raison de la perpétuation de plusieurs niveaux de fractures numériques. Ainsi, même s'il est bien sûr envisageable que des évolutions puissent avoir lieu dans le futur, ces ajustements des discours restent à la marge et ne semblent pas de nature à permettre de sortir de cette spirale d'une répétition inopérante de l'injonction à la CSTI pour tous.

\subsection{De multiples résultats individuels et locaux, mais toujours insuffisants}

Pourtant, du point de vue de leurs différents protagonistes, ces dispositifs, programmes et actions se sont révélés en grande majorité pertinents à l'échelle locale ou thématique, atteignant leurs objectifs pour les personnes ou les groupes concernés : de multiples traces de ces efficacités à l'échelle microsociale ont été observées, sous forme de trajectoires de personnes, d'audience d'évènements ou de fréquentation de lieux spécialisés (OPECST, 2014). En revanche, le bât blesse lorsque que l'on change d'échelle d'observation: force est de constater que ces programmes et dispositifs n'ont pas changé la situation au niveau macrosocial, comme le démontre justement au premier chef cette vaine répétition des discours d'experts ou de politiques. Ainsi, même les grands dispositifs de masse se sont montrés impuissants à enrayer la déploration de la présumée «désaffection »: cela vaut globalement, pour tous les dispositifs nationaux de CSTI, comme la Cité des sciences ou de l'industrie, comme les réseaux de CCSTI, comme les vingtaines d'éditions d'opérations nationales du type «Fête de la science » ou « Nuits

filières générales de l'enseignement supérieure et un présumé désamour des sciences.

${ }^{4}$ A noter tout de même que l'année 2014 a vu aussi la production par le haut conseil à la science et technologie (HCST), d'ailleurs non coordonnée avec celui de l'OPECST, d'un rapport beaucoup plus spécialisé sur l'attractivité, qui traite en particulier des questions de valorisation du doctorat (HCST et CEREQ, 2014), disponible sur : http://pmb.cereq.fr/doc num.php?explnum_id=1236 (consulté le 27/03/2016) 
des étoiles ». L'addition de tous ces efforts, malgré leur pertinence indéniable vis-à-vis de leurs nombreux bénéficiaires directs, n'a réussi en rien à rendre caducs ces discours, ni même à les pondérer (Labasse 1999 ; Schiele, 2005 ; Las Vergnas, 2011a).

\subsection{Une CSTI impuissante à changer les représentations sociales de la science}

Que montrent alors plus précisément ces répétitions systématiques de constats d'échec malgré cette pertinence locale ? Que l'impuissance à l'échelle sociale des politiques de CSTI n'est pas due à des difficultés cognitives individuelles, puisque de multiples expériences démontrent que celles-ci sont surmontables dès lors qu'il y a motivation à l'appropriation de savoirs, mais qu'elle a à voir avec d'autres mécanismes, caractéristiques eux de l'échelle macrosociale. En effet, si à l'échelle individuelle ou des petits groupes, la question de la CSTI «pour tous » renvoie à celle de la pédagogie, des apprentissages et des obstacles cognitifs bachelardiens, à l'échelle de la société ce sont d'autres mécanismes majeurs (socio-économiques en particulier) qui sont l'œuvre et qui déterminent bien plus les représentations sociales.

On peut bien sûr penser que la répétition des discours est liée à leurs ambiguittés sémantiques déjà signalées plus haut et en particulier au caractère d'injonction prophétique floue du projet de la «CSTI pour tous » (Schiele, 2005, Las Vergnas, 2011b): ambiguïté de la terminologie de « culture » employée à la fois pour désigner le développement culturel individuel, mais aussi au sens figuré pour désigner des pratiques culturelles (visite de musées, lecture ou consultation d'ouvrages imprimés ou multimédias), naïveté d'une approche non spécifiée par public ou par âge, manque de clarification des objectifs sociaux visés, absence de définition du périmètre de la STI, (amalgame simpliste entre science, technique et industrie, malgré le pluriel utilisé aujourd'hui pour désigner «les cultures»), sans oublier une confusion entre médiation « cognitive » par appropriation de savoir et médiation «idéologique » par un dialogue entre opinions profanes et savoirs des scientifiques.

Cependant, au-delà de ces approximations sémantiques et idéologiques, la répétition sur quatre décennies donne à penser que la «CSTI pour tous » se heurte à un obstacle systématique : un invariant socio-culturel ou socio-éducatif se perpétuerait qui inhiberait toute transformation sociétale en la matière, malgré les succès locaux. 


\subsection{Un rapport aux sciences construit très majoritairement dans l'enseignement initial}

Voilà qui nous conduit à nous intéresser à l'organisation du système scolaire puisque c'est avant tout en son sein que se construit le rapport aux savoirs dits scientifiques pour les non spécialistes: la plupart des personnes ne feront jamais autant d'activités dénommées officiellement «sciences » que durant leur scolarité obligatoire. De fait, durant son enseignement primaire ou secondaire, toute personne scolarisée en France, aura consacré au moins un gros millier d'heures à suivre des cours dédiés aux disciplines qualifiées de «scientifiques». En effet, même ceux qui quittent les études à la fin de la troisième auront suivi au moins 240 semaines de cours [30 semaines chacune des 8 années entre le CE1 et la 3eme] où leur en auront été prodiguées en moyenne 5 heures de sciences scolaires par semaine. Sil'on ajoute la seconde, la 1ere et les devoirs à la maison, on est même plus près, pour la majorité de la population, des deux milliers d'heures. En comparaison, ne consacrent aux sciences un volume horaire (non scolaire) équivalent que les quelques $15 \%$ de la population nationale que l'on peut considérer comme des professionnel(le)s du travail scientifique ${ }^{5}$... ou les rares personnes qui dans leur vie consacreront plus de $1000 \mathrm{~h}$ à s'approprier de la vulgarisation.

\subsection{Une efficacité sociale plombée par l'invariant majeur qu'est la «catégorisation scolaire »}

Dans le système éducatif français ${ }^{6}$, l'enseignement secondaire des disciplines dites scientifiques est marqué par quatre caractéristiques, sachant que dans le secondaire le terme «scientifique » désigne les maths, les SVT et la physique et exclut les $\mathrm{SHS}^{7}$ :

1) l'organisation des filières générales oblige les adolescent(e)s à se déterminer (ou à être orientés) entre trois séries distinctes pour préparer un baccalauréat général : soit scientifique, soit

\footnotetext{
${ }^{5} 15 \%$ max de la population nationale de chercheurs, ingénieurs, médecins ... technologues. Et seulement quelques centaines de milliers de membres de clubs qualifiables de scientifiques, tous profils confondus.

${ }^{6}$ Comme celui de nombreux pays (voir le caractère international de l'échelle d'anxiété en mathématique promue par «Adult learning Maths, cf plus loin), mais sans doute plus encore : On peut en effet postuler que cette catégorisation étant moins marquée au Royaume Uni (avec un système moins clivant que le Bac $\mathrm{S}$ au niveau de la fin des études secondaires) au Bac) ou dans les pays tenants du dual system de l'alternance et des hautes écoles (qui séparent moins technologies et sciences fondamentales dans leurs filières). Malheureusement sur cette question spécifique des effets de la catégorisation scolaire nous ne disposons pas vraiment d'études comparatives. A signaler cependant la thèse en cours de A. Temkeng (en cours) sur les adultes reprenant des études supérieures au Cameroun et qui comporte une enquête différentielle sur ce sujet entre les zones d'influence françaises et anglaises.

${ }^{7}$ Pour être tout à fait précis, il faut noter que les bacs technologiques mentionnent aujourd'hui tous dans leur dénomination extensive «sciences de ... », mais aucune ambiguïté n'est perçue quand on parle de filière scientifique. Ou de « séries à caractère scientifique » pour désigner ensemble S, STI2D et STL (RERS, 2015 p 110)
} 
littéraire, soit économique et sociale (S, L et ES) ${ }^{8}$.

2) la volumétrie de la filière «scientifique » aboutit à ce que 17 à 20\% (17\% jusqu'en 2010 et en progression depuis jusqu'à $20 \%$ actuellement) de chaque classe d'âge obtiennent le bac général scientifique $(21 \%$ à $25 \%$ si on y ajoute les bacs technologiques dits «à dominante scientifique »). La figure 1 rend compte de cette répartition des flux d'élèves dans l'enseignement secondaire en France vis-à-vis des matières dites scientifiques (source des données : RERS, 2014).

3) l'image de cette filière $\mathrm{S}$ est devenue celle d'une «vraie série générale, ouvrant de très larges débouchés, y compris dans le domaine des sciences humaines, du droit et des sciences politiques [...en raison de la ] substitution progressive d'une logique de hiérarchisation des séries, privilégiant à l'excès la série scientifique, à une offre plurielle de formation générale, fondée sur une différenciation des aptitudes et conduisant de bons et de moins bons élèves à des études supérieures distinctes. » (IGEN et IGAEN, 2006, p 92-93),

4) la détermination des élèves vers d'autres filières que la filière générale dite «scientifique » s'effectue aujourd'hui majoritairement sur la base de notes progressivement jugées insuffisantes en maths au fur et à mesure des années du collège, à partir d'un vivier alimenté par les effets des représentations qu'ont les adultes des filières scientifiques puisque elle est perçue comme la filière qui offre le plus de débouchés (cf. point précédent).

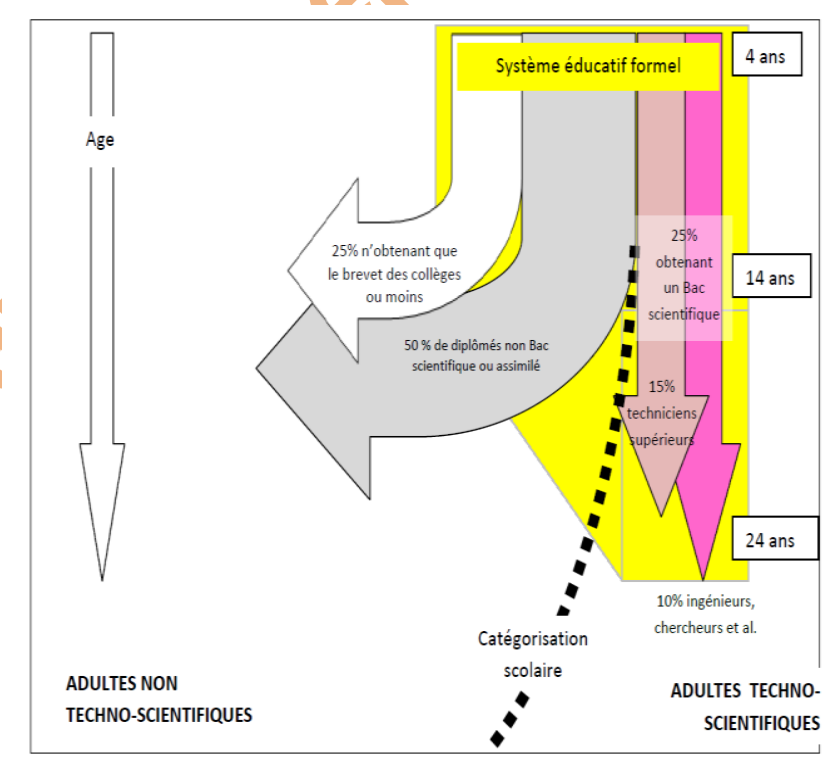

Figure 1 : répartition des flux vis-à-vis des matières dites scientifiques dans le secondaire en France (source des données : RERS, 2014)

\footnotetext{
${ }^{8}$ ce qui dans les faits empêchera par la suite le retour des non bacheliers S vers des études de sciences « dures » ou technologiques.
} 
Il résulte de cet ensemble de facteurs que la détermination vers une autre filière que S (ou STL et STI2D) est vécue pour la majorité des jeunes (et de leurs proches) comme un constat d'incompétence vis-à-vis des «sciences » au sens scolaire du terme. Ainsi, pour une grande partie des non orientés vers le Bac S (et reçus), les obstacles cognitifs (Bachelard, 1938) sont-ils doublés de deux autres, liés à cette sanction d'incompétence, qui, dans des travaux antérieurs (Las Vergnas, 2011a), ont été respectivement qualifiés, de « conatif» et « scholastique ».

L'« obstacle conatif » exprime le fait que des individus se sentent incapables de traiter d'une question de nature scientifique, à cause de la sanction d'inaptitude reçue lors de leur vécu scolaire antérieur. En référence au «sentiment d'efficacité personnelle» (SEP) introduit par Bandura (2003), on peut émettre l'hypothèse que l'obstacle conatif s'apparente à un sentiment d'inefficacité personnelle vis-à-vis de ce qu'on a appris à appeler «sciences » au collège ou lycée et plusieurs auteurs ont montré l'existence d'obstacles affectifs (Baruk, 1973 ; Nimier, 1988). Cet obstacle est d'ailleurs loin d'être une spécificité française et de nombreux auteurs réunis en particulier au sein d'un réseau international intitulé «Adults Learning Mathematics » utilisent une échelle «d'anxiété en mathématique » (Suinn et Winston, 2003) et convergent vers le constat résumé par Klinger (2006, p 165-166, traduction personnelle): «Les conceptions mathématiques peuvent être fortement influencées par les expériences de l'école primaire et les attitudes des parents; de premières expériences d'apprentissage traumatisantes étant susceptibles d'exercer un effet à long terme de sorte que beaucoup se perçoivent dès un âge précoce comme non-matheux ce qui conduit à une absence de motivation. ». Ce dernier a montré que les attitudes négatives, le manque de SEP et l'anxiété mathématique des adultes sont «fortement influencées par les expériences d'apprentissage précoce ». De cet obstacle conatif découlent en particulier les difficultés systématiques de la majorité des non Bac $\mathrm{S}$ à envisager d'utiliser tout formalisme algébrique même rudimentaire dans leur vie quotidienne.

L' 《obstacle scolastique » quant à lui permet de décrire le fait que la « relation à la science » d'une activité conduite par la personne est masquée à celle-ci, scotomisée ; cet obstacle apparait chez des adultes pour qui la qualification de «scientifique » ne peut être attribuée qu'à ce qui leur rappelle les disciplines scientifiques scolaires. Cet obstacle n'inhibe donc pas la conduite de l'activité, mais empêche l'individu de voir que certains des résultats positifs qu'il obtient ont un rapport avec des capacités qualifiables de « scientifiques ». 
La production systématique de ces obstacles individuels chez les personnes, laisse penser que s'installe également à l'échelle sociale la représentation d'un monde dans lequel les individus sont soit «scientifiques» soit «non scientifiques». Cette représentation d'un clivage, d'une frontière quasi-irréversible et déterministe laisse penser que l'on sera -à vie- d'un côté ou de l'autre, renvoyant à l'image d'Épinal d'une «bosse des maths » présente chez certains et absente chez d'autres. Au vu du poids du Bac $\mathrm{S}$ dans le système de sélection de l'enseignement scolaire, nous appelons ce phénomène la «catégorisation scientifique scolaire » (CSS).

En fait peu d'études existent précisément sur ce sujet. Une enquète Universcience/Credoc sur la population générale (Bigot \& al. 2013) montre néanmoins qu'à la question de savoir si des personnes aujourd'hui adultes se considéraient plutôt « littéraires » ou « scientifiques » ou «ni scientifiques, ni littéraires » durant leur scolarité (p. 11), les personnes se répartissent en trois grandes catégories : littéraire (29\%), scientifique (26\%) et ni l'un ni l'autre (36\%) tandis que seulement $8 \%$ se considéraient comme « autant l'un que l'autre » et que la question n'obtient que $1 \%$ de «non réponse $»$. De plus :

"Parmi [les 36\% de] personnes interrogées [qui se sont] considérées «ni scientifiques, ni littéraires »[,] cette catégorisation des compétences leur rappelle qu'elles «n'étaient pas faites 》 pour l'école (selon leurs propres termes). Elles expriment d'ailleurs un ensemble d'idées négatives sur l'école : un désintérêt pour les matières scientifiques et littéraires ("je ne m'intéresse à aucun de ces sujets »), un rejet de l'école (" déjà, moi, l'école ce n'était pas trop mon truc ») ou encore un sentiment de ne pas être doué pour ces matières (" je n'étais pas bonne à l'école »). »(p. 12).

Bien sûr, l'étude devra être reprise en posant la spécifiquement la question de savoir si les personnes «scientifique ou non scientifique ? », plutôt que de se focaliser sur l'opposition trop réductrice et scolastique entre «scientifiques et littéraires », mais on peut déjà constater qu'avec le même échantillon on aurait -selon toute vraisemblance- obtenu toujours $26 \%$ de personnes répondant qu'elles se considéraient comme purement «scientifiques », 8\% qui se pensaient «littéraires et scientifiques » donc entre autres « scientifiques » (soit 26+8=34\% en partie ou en totalité «scientifiques »), ce qui laisse ainsi $65 \%(29 \%+36 \%)$ de «non scientifiques ». Autre enseignement de l'étude (p. 12), parmi les 36\% de «ni l'un, ni l'autre », seuls $8 \%$ (soit 3\% de la totalité de l'échantillon interrogé) rejettent la pertinence d'une telle catégorisation S/L. Ainsi, en ce qui concerne le rejet de la catégorisation des «scientifiques », on pourrait faire l'hypothèse qu'il se situerait entre $4 \%$ (3\% de rejet et $1 \%$ de non réponses) et $12 \%$ si l'on considérait nécessaire d'y ajouter les $8 \%$ de personnes ayant répondu « les deux ». 
Le fait que ce rejet de la question et de la catégorisation semble concerner si peu de personnes (entre $4 \%$ et $12 \%$ ) confirme l'idée que cette $\operatorname{CSS}^{9}$ est bien un fait social total.

\subsection{Inutile d'espérer développer la CSI pour tous les adultes sans réduire cette catégorisation}

Entretenant l'illusion de l'amélioration simultanée de la détection de l'élite et du partage des savoirs par tous, les discours officiels de CSTI pour tous oublient que cette CSTI se heurte à ce rôle sélectif du système éducatif initial, non seulement au niveau individuel, mais aussi au niveau sociétal par la construction de ce clivage découlant de la CSS. Or, dès lors que l'on s'intéresse à débusquer ou à prévenir une présumée « désaffection des sciences » on ne peut nier que l'on est là face à un phénomène social partagé massivement qui va bien au-delà de perceptions dont chacun porterait la responsabilité. Ainsi, au-delà des obstacles individuels vis-à-vis des savoirs scientifiques qui eux peuvent être surmontés localement au cas par cas, les injonctions nationales à la «CSTI pour tous » se heurtent systématiquement au clivage résultant de cette fonction de catégorisation de l'enseignement secondaire.

\section{Une typologie des actions et programmes de CSTI en référence à CSS}

\subsection{Comment peut-on décrire les différents types d'objectifs des actions de CSTI ?}

Dans des travaux antérieurs (Las Vergnas 2011a), nous avons proposé une typologie des actions de CSTI organisée en fonction de leur positionnement vis-à-vis de la CSS. Cette typologie s'organise en cinq catégories que la figure 2 présente et place par rapport au diagramme des flux scolaires emprunté à la figure 1 .

\footnotetext{
${ }^{9}$ On peut bien sûr considérer que la plupart des filières produisent de telles catégorisations dans d'autres domaines (les «musiciens », les « sportif s», ce qui peut être vu comme une simple résultante de l'organisation par diplôme. En revanche c'est le poids social particulier qui ajouté à ce mécanisme par le fait qu'il s'agit ici de la filière d'excellence, qui fait de ce mécanisme de catégorisation laisse des séquelles à la plupart des adultes non sélectionnés par ce clivage. (IGEN \& IGAEN, 2005)
} 


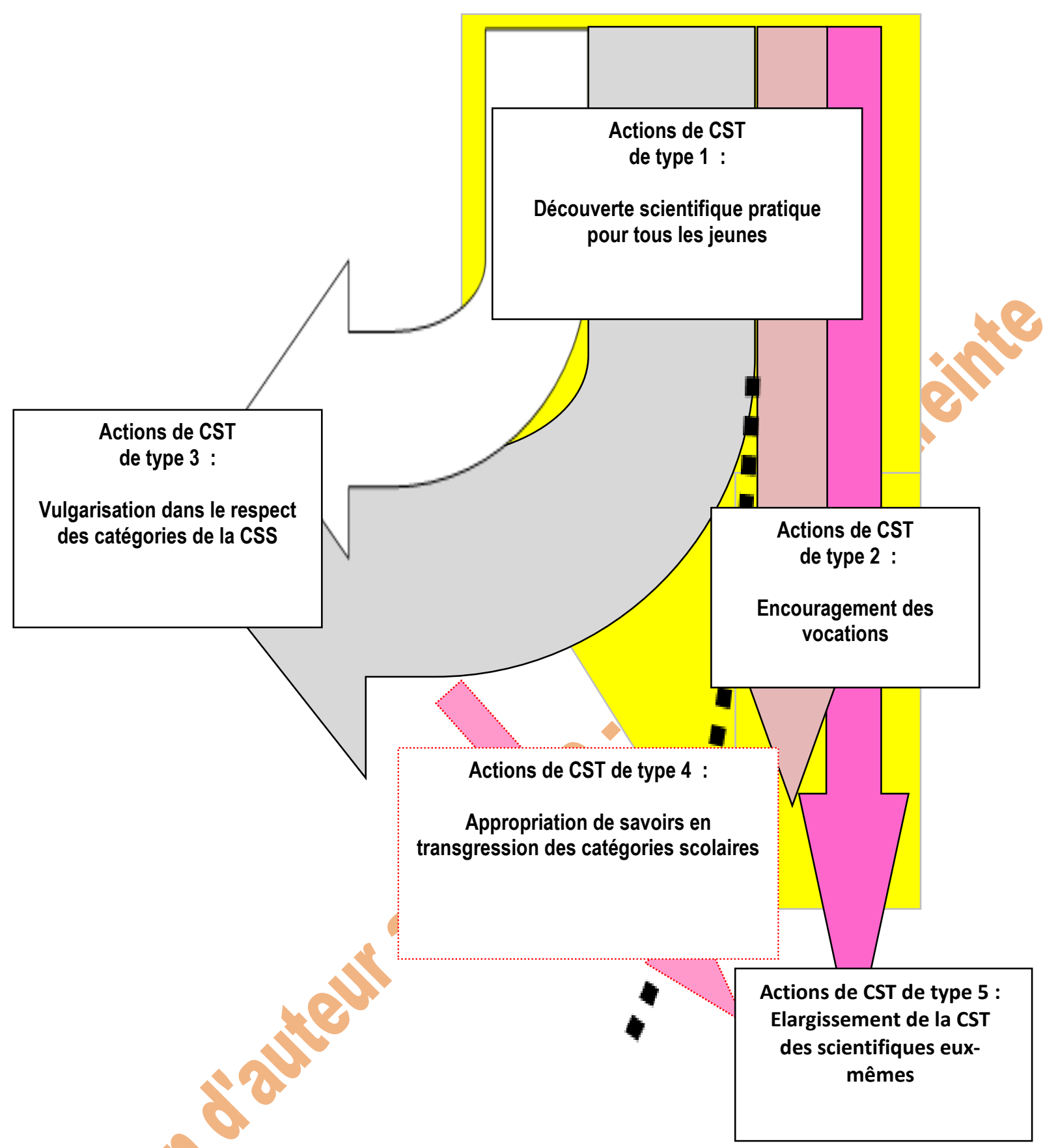

Figure 2 : actions et dispositifs de CSTI

positionnées par rapport à la catégorisation scientifique scolaire 
On retrouve ces cinq catégories détaillées dans le tableau T1.

\begin{tabular}{|c|c|c|c|c|c|c|c|}
\hline type & Intitulé & $\begin{array}{l}\text { Exemples d'activités } \\
\text { des personnes }\end{array}$ & Age concerné & $\begin{array}{c}\text { Exemples de } \\
\text { dynamiseurs de ce } \\
\text { type de dispositifs }\end{array}$ & $\begin{array}{l}\text { Catégories de } \\
\text { publics }\end{array}$ & Effet social visé & $\begin{array}{c}\text { Effet } \\
\text { individuel } \\
\text { visé }\end{array}$ \\
\hline $\begin{array}{c}\text { type } \\
1\end{array}$ & $\begin{array}{c}\text { Découverte scientifique } \\
\text { pratique } \\
\text { pour tous les jeunes }\end{array}$ & $\begin{array}{l}\text { Visite de la « Cité } \\
\text { des enfants » }\end{array}$ & $\begin{array}{c}\text { Antérieur à la } \\
\text { CSS }\end{array}$ & $\begin{array}{l}\text { Médiateurs } \\
\text { scientifiques, } \\
\text { enseignants }\end{array}$ & $\begin{array}{l}\text { Tous les jeunes } \\
\text { publics }\end{array}$ & $\begin{array}{l}\text { Compense les } \\
\text { problèmes de } \\
\text { formation des } \\
\text { professeurs des } \\
\text { écoles } \\
\end{array}$ & $\begin{array}{l}\text { Brassage } \\
\text { social } \\
\text { prévenant } \\
\text { la CSS }\end{array}$ \\
\hline $\begin{array}{l}\text { type } \\
2\end{array}$ & $\begin{array}{l}\text { Encouragement des } \\
\text { vocations }\end{array}$ & $\begin{array}{c}\text { Participation aux } \\
\text { Olympiades de } \\
\text { chimie }\end{array}$ & $\begin{array}{c}\text { Postérieur à la } \\
\text { CSS }\end{array}$ & $\begin{array}{c}\text { Médiateurs, } \\
\text { enseignants et } \\
\text { chercheurs }\end{array}$ & $\begin{array}{l}\text { "Scientifiques" } \\
\text { au sens scolaire }\end{array}$ & $\begin{array}{l}\text { Peut réduire les } \\
\text { discriminations }\end{array}$ & $\begin{array}{l}\text { Confirme } \\
\text { la CSS }\end{array}$ \\
\hline $\begin{array}{l}\text { type } \\
3\end{array}$ & $\begin{array}{l}\text { Vulgarisation dans le } \\
\text { respect des catégories }\end{array}$ & $\begin{array}{c}\text { Ecoute de } \\
\text { conférences, } \\
\text { documentaires à la } \\
\text { TV }\end{array}$ & $\begin{array}{c}\text { Postérieur à la } \\
\text { CSS }\end{array}$ & $\begin{array}{l}\text { Vulgarisateurs, } \\
\text { éditeurs, } \\
\text { chercheurs, } \\
\text { journalistes }\end{array}$ & $\begin{array}{l}\text { "Scientifiques » } \\
\text { au sens scolaire } \\
\text { ou non }\end{array}$ & $\begin{array}{c}\text { Favorise le dialogue } \\
\text { entre chercheurs et } \\
\text { non chercheurs }\end{array}$ & $\begin{array}{l}\text { Sans effet } \\
\text { sur la CSS }\end{array}$ \\
\hline $\begin{array}{l}\text { type } \\
4\end{array}$ & $\begin{array}{l}\text { Appropriation de savoirs } \\
\text { en transgression des } \\
\text { catégories scolaires }\end{array}$ & $\begin{array}{l}\text { Recherche amateur } \\
\text { d'astéroïdes, } \\
\text { auto-clinique } \\
\text { médicale }\end{array}$ & $\begin{array}{c}\text { Postérieur à la } \\
\text { CSS }\end{array}$ & $\begin{array}{l}\text { Auto-portage, } \\
\text { réseaux sociaux } \\
\text { entre pairs, acteurs } \\
\text { de l'educ. Pop. }\end{array}$ & $\begin{array}{c}\text { Non } \\
\text { « scientifiques » } \\
\text { au sens scolaire } \\
\text { Non scientifiques } \\
\text { scolaires }\end{array}$ & $\begin{array}{l}\text { Réduit au cas par } \\
\text { cas la CSS avec } \\
\text { des DP }\end{array}$ & $\begin{array}{c}\text { Dépasse } \\
\text { localement } \\
\text { les } \\
\text { obstacles } \\
\text { liés à la } \\
\text { CSS }\end{array}$ \\
\hline type 5 & $\begin{array}{c}\text { Elargissement de la CST } \\
\text { des scientifiques eux- } \\
\text { mêmes }\end{array}$ & $\begin{array}{l}\text { Lecture de la revue } \\
\text { La Recherche }\end{array}$ & $\begin{array}{l}\text { Largement } \\
\text { postérieur à la } \\
\text { CSS }\end{array}$ & $\begin{array}{c}\text { Chercheurs, } \\
\text { associations et } \\
\text { médias scientifiques }\end{array}$ & $\begin{array}{l}\text { "Scientifiques" } \\
\text { au sens scolaire }\end{array}$ & $\begin{array}{c}\text { Améliore l'ouverture } \\
\text { transversale des } \\
\text { chercheurs }\end{array}$ & $\begin{array}{l}\text { Confirme } \\
\text { la CSS }\end{array}$ \\
\hline
\end{tabular}

Tableau T1 : typologie des actions et dispositifs de CSTI en relation à la catégorisation scientifique scolaire

Le premier type regroupe les actions et dispositifs qui s'adressent aux jeunes non encore confrontés à la CSS, afin de leur permettre à tous une découverte scientifique pratique. Ces actions et dispositifs ont une double utilité sociale qui a été repérée et étudiée par de nombreux auteurs : compenser le manque d'enseignement de découverte expérimentale des sciences au primaire et au collège, pallier le manque de formation scientifique des professeurs des écoles.

Le deuxième type concerne des actions qui visent les jeunes aux alentours de l'âge de la CSS (à partir de 13 ou 14 ans), et qui se définissent par leurs objectifs d'encouragement des vocations. C'est notamment le cas des opérations «Faites de la science » qui cherchent en particulier à susciter leur curiosité et leur enthousiasme pour les études scientifiques, ou des olympiades de maths, de physique ou de chimie. Puisqu'elles ne sont pas en mesure d'augmenter les places disponibles dans les filières scientifiques, il s'agit d'opérations à somme nulle, qui peuvent être perçues comme servant à brasser les types de candidats (en termes de sexe, de CSP des parents, de lieu de résidence) ou à s'assurer qu'il y aura suffisamment de pression sur les places à pourvoir pour s'assurer des motivations, voire de la pertinence des orientations. 


\subsection{Regarder la CSTI pour adultes selon trois types, par référence à la CSS.}

Qu'en est-il pour les adultes, c'est à dire pour les personnes ayant dépassées la période de la CSS initiale ? Hormis l'élargissement de la CSTI des scientifiques eux-mêmes (type 5) que l'on peut considérer comme une suite de l'encouragement des vocations et une animation de la cohérence de la CSS en assurant au moins un minimum de brassage des savoirs entre ceux qui sont du bon côté de la CSS), si l'on prend comme analyseur la CSS on peut considérer que deux familles de pratiques de CSTI (type 3) et (type 4) coexistent.

Par définition, ces deux familles correspondent à deux façons de se positionner vis à vis de la CSS. Le type 3 favorise une communication, voire un dialogue entre les professionnels de la recherche scientifique ${ }^{10}$ et les profanes, sans remettre en cause le clivage correspondant à la CSS, tandis que le type 4 vise à permettre le dépassement voire la transgression de la CSS par l'adoption d'une posture caractéristique de l'investigation scientifique. Concrètement, les actions qui permettent de mieux comprendre le travail de tel ou tel scientifique au travers d'une émission ou d'une conférence décrivant son dernier ouvrage de vulgarisation correspondent au type 3 ; la mise en place d'un projet d'observation des phénomènes mutuels d'occultations des satellites de Jupiter s'inscrit dans le type 4. On peut aussi prendre comme critère discriminant entre type 3 et type 4 la question de savoir qui essaie de se mettre au niveau de qui : Dans une situation de type 3 c'est le scientifique scolairement diplômé qui essaie de se mettre au niveau de ceux qui sont de l'autre côté de la CSS. Dans le type 4, ce sont les non scientifiques qui tentent de se mettre au niveau d'une activité scolairement pensée comme de l'autre côté de la CSS.

\subsection{Type 3 : organiser le dialogue et non réduire la catégorisation}

A contrario de celles de type 4, les situations de type 3 ne font donc qu'instituer durablement la CSS. Pour autant, ces actions de type 3 ne sont pas jugées contre-productives aux yeux de tous les prescripteurs de la CSTI. De fait, la grande majorité des actions qui «vulgarisent» au sens premier du terme sont vues comme favorisant la compréhension du rôle et de l'intérêt des professionnels de la recherche à défaut de favoriser la réduction de la CSS (Bensaude-Vincent, 2010 ; Felt 2010) et correspondent au type 3.

\footnotetext{
${ }^{10}$ Pour beaucoup d'activités de CSTI, le mot science est en fait utilisé pour désigner les activités institutionnalisées de recherche scientifique.
} 
En effet, on observe que les politiques de CSTI pour adultes sont en train d'être plus orientées vers l'organisation d'un dialogue entre des Scientifiques et les non-scientifiques que vers la lutte contre la CSS : les feuilles de route des financeurs sont de bâtir un lien entre la science et la société. Or, en dehors de certaines activités spécifiques de «sciences participatives » sur lesquelles nous reviendront à propos du type 4 , ces programmes pour adultes de sciences dans la société instaurent et gèrent plus des dialogues entre les opinions des non-scientifiques et les connaissances scientifiques, que des partages de savoirs.

\subsection{Type 4 : permettre a posteriori une transgression de la catégorisation scolaire}

Le type 4 correspond par définition à des dispositifs permettant l'appropriation de savoirs scientifiques et de méthodes en s'autorisant à transgresser les limites de la CSS initiale. A priori, ce type devrait comprendre au premier chef tous les dispositifs de formation permanente permettant l'obtention tardive d'au moins un équivalent du Bac technoscientifique, par une reprise d'études, l'obtention du diplôme d'accès aux études universitaires (DAEU) option sciences, ou encore une validation des acquis de l'expérience (VAE) à contenu scientifique, sous-ensemble que l'on pourrait donc qualifier de Type 4 «diplômant ». Cependant, force est de constater que de telles activités et pratiques ne sont que très rarement regardées comme faisant partie du champ de la CSTI, de la même façon que l'enseignement formel n'est pas considéré comme entrant dans le spectre de la CSTI : vus comme relevant du champ de la «formation tout au long de la vie », ces DAEU ou VAE ne sont quasiment jamais rattachés dans la littérature et dans les discours à de l'action culturelle. Ce constat renvoie à ce qui avait déjà été observé par Ackermann et Dulong dès 1971 lorsqu'ils notaient, dans un article de la «revue française de sociologie » intitulée « un nouveau domaine de recherche : la diffusion des connaissances scientifiques », l'incompatibilité de l'approche centrée sur les compétences de l'éducation permanente avec celle centrée sur la vulgarisation qui, selon les auteurs, «apparait comme un phénomène où la transmission de connaissances joue un rôle minime ».

Il y a néanmoins d'autres dispositifs et pratiques correspondant à une vision plus habituelle de la CSTI qui entrent dans le type 4 : par exemple, ceux qui peuvent conduire à une reconnaissance comme scientifique amateur, au premier rang desquels on pense aux sciences participatives ou collaboratives, au sens des pratiques d'implication de volontaires profanes comme cochercheurs ; c'est aussi le cas des recherches pragmatiques conduites par des personnes touchées 
par un problème personnel ou communautaire (comme des problèmes de pollution, de risque alimentaire ou de défense de la biodiversité) les conduisant à mener des investigations à dimension scientifique, comme celles menées par des malades chroniques (Jouet \& al., 2014) ou celles que Phil. Brown (1987) a désigné sous le nom d'épidémiologie populaire.

\subsection{Penser la transgression oblige à questionner la définition de la Science}

Au plan épistémologique, l'analyse du type 4 non «diplômant » ne peut se faire sans poser la question d'une définition autre que scolaire de ce qui «fait science» ou mérite d'être appelé ainsi, sachant que cette question de définition du caractère scientifique est toujours au cœur des questions de CSTI. Dans le début du présent texte, l'approche de la « CSTI pour tous » par le fait social total que constitue la CSS permet de se raccrocher à une vision stable de la science qui est celle transmise par l'enseignement général secondaire français, à savoir la science comme un corpus de savoirs liés aux disciplines mathématiques, physiques et des SVT. En revanche dès lors que l'on cherche à définir la transgression de la CSS, il devient nécessaire d'examiner d'autres définitions du caractère scientifique. La question est bien sûr multiple. Elle peut se poser tant en termes de champs disciplinaires (inclut-on ou non les SHS ?) qu'en termes de rapport aux technologies et aux applications (considère-t-on les technosciences ?), voire en termes de critères de scientificité.

Aussi, le point de vue naïf selon lequel le rôle des acteurs de la CSTI devrait être de chercher à rendre au mieux compte d'un fait social qui serait une «Science » définie de manière univoque n'est pas tenable : de fait, ce qui est appelé « Science » n'est qu'un construit social fluctuant dont les principaux fabricants sont le système éducatif et les médias, parmi lesquels les acteurs de la CSTI sont au premier rang. Ainsi, par exemple, la question de la fidélité de la représentation d'une telle «Science » par les acteurs de la CSTI n'a pas de sens autre qu'en grande partie autoréférent; d'autant que l'on peut déjà s'interroger sur la cohérence entre les différentes représentations sociales diffusées par le système scolaire, qui, même si elles se rattachent toutes à des corpus disciplinaires, n'incluent pas les mêmes disciplines (en particulier en ce qui concerne les sciences humaines et sociales) selon qu'il s'agit des enseignements secondaires général, technologique, professionnel ou de l'enseignement supérieur.

Dans des travaux antérieurs (Las Vergnas, 2016), nous avons constaté qu'en plus de ces représentations -véhiculées par l'enseignement scolaire- des sciences comme des assemblages de 
corpus disciplinaires coexistent trois autres familles de représentations auxquelles se rattachent ou se réfèrent, selon les contextes et les interlocuteurs, les différents acteurs de la CSTI. On peut considérer que les deux premières correspondent respectivement à la Little Science et à la Big Science tels que ces deux régimes ont été définis et opposés par Derek Price, dans son ouvrage justement intitulé Little Science, Big Science (1963) : Price y expose la différence entre une conception contemporaine d'une science impensable sans gros investissements internationaux, sans commune mesure avec la science à dimension humaine des XVII ème et XVIII ème siècles. De fait dans les activités de CSTI d'aujourd'hui on retrouve aussi bien des clubs ou ateliers périscolaires qui s'appuient sur la représentation de la Little Science pour s'intituler « scientifiques » alors que des cafés des sciences ou des documentaires vont insister au contraire sur le caractère incontournable de la mondialisation pour toute activité dite scientifique aujourd'hui. Par ailleurs, le développement d'approche de sciences participatives ou collaboratives avec des programmes emblématiques comme les PICRI ${ }^{11}$ se réfère à un troisième régime, celui de l'Impure Science, tel qu'il a été défini par Steven Epstein dans son ouvrage éponyme (1996) pour désigner les incursions des malades du SIDA dans la clinique de leur maladie.

Le fait d'être rattaché implicitement ou explicitement à telle ou telle représentation de la science change radicalement le point de vue sur la possibilité ou non de créer des dispositifs de transgression de la CSS : à chacune de ces représentations de la science correspond un projet politique de la CSTI pour adultes et des volontés plus ou moins fortes de développer plutôt des actions de type 3 ou 4 .

Ainsi, pour des acteurs prônant comme seules représentations de la science la maitrise des corpus disciplinaires scolaires, ne peuvent exister comme dispositifs de CSTI de type 4 que les reprises d'études et assimilés, donc uniquement ceux qui sont diplômant. Voilà qui va de pair avec le fait que pour beaucoup de décideurs politiques et de financeurs, faire œuvre de CSTI signifie valoriser la recherche scientifique institutionnalisée et que perdure une confusion entre «Science » et organismes de recherche, conduisant presque exclusivement à de la production de CSTI de type 3, la plus apte à en assurer la communication ou l'outreach pour en reprendre la dénomination donnée par les instances européennes.

\footnotetext{
${ }^{11}$ PICRI : Partenariats institutions-citoyens pour la recherche et l'innovation (Picri). Il s'agit de programmes de recherche reposant sur une collaboration étroite entre laboratoires de recherche publics et organisations issues de la société civile, voir par exemple http://www.iledefrance.fr/competence/picri
} 
A contrario, pour des acteurs issus des courants de l'éducation populaire, la science doit être vue comme devant rester une démarche accessible à tous : en effet les tenants de ce point de vue prônent l'émancipation des personnes par le développement de «l'esprit scientifique », c'est-àdire par la mise en pratique de démarches d'investigation d'inspiration expérimentale. Pour permettre à leur point de vue de se déployer, ils soutiennent que le développement actuel et mondialisé de la Big Science ne doit pas conduire à exclure du champ considéré comme scientifique des pratiques réflexives plus individuelles ou locales qui ont nourri la Little Science, d'autant qu'elles vont de pair avec celles d'Impure Science qui reconnaissent une place aux profanes comme coproducteurs de savoirs. Ainsi, selon ces militants de l'éducation populaire, il est tout à fait légitime de considérer que la réflexivité et les phénoménologies pragmatiques de chacun méritent d'être vue comme une forme scientifique dès lors qu'elles s'organisent autour de la recherche de faits, de preuves et de confrontations sceptiques.

\section{Est-il actuellement envisageable de réduire la CSS ?}

\subsection{La CSS a toujours été un point aveugle des discours sur la CSTI}

Les promoteurs d'actions de type 4 ont beau affirmer qu'ils développent des opportunités de transgresser la CSS, cette transgression n'est valide qu'aux yeux de ceux qui veulent bien la reconnaitre. Or, du point de vue dominant aujourd'hui des sciences scolaires et de la Big Science, ce ne sont pas les pratiques des personnes qui sont scientifiques ou non : ce sont leurs statuts, marqués par un diplôme et un métier. Se pose alors la question de savoir s’il serait envisageable de réduire la CSS en elle-même en tant que création de l'enseignement secondaire de CSS plutôt que de chercher à en favoriser la transgression a posteriori. Sur des dernières décennies, la revue de littérature montre que cette question est quasiment absente des discours et des programmes politiques. Régulièrement, quelques auteurs, suivant en cela Jean-Marc Levy Leblond (voir par exemple Levy-Leblond, 2004), citent néanmoins le petit texte fondateur de Charles Snow (1959) dénonçant la fracture entre les «Two Cultures »), mais force est de constater que la nécessité de réduire le clivage tel qui est spécifiquement produit au lycée par le baccalauréat $S$ n'est pas identifié comme un objectif. . Certes, le monde scolaire secondaire et primaire évolue d'année en année, tentant justement de rendre les sciences scolaires moins abstraites et de s'assurer de leur enseignement à tous les élèves, en les réinscrivant dès les programmes du primaire, en en 
affirmant l'importance dans le socle commun de connaissances et de compétences ${ }^{12}$ et en promouvant la pratique de l'interdisciplinarité ${ }^{13}$.

\subsection{La réduction de la CSS au lycée n'est toujours pas considérée comme prioritaire}

Pour préciser la situation actuelle, nous avons analysé les préconisations du dernier rapport de l'OPECST (2014) et avons cherché parmi les 79 recommandations édictées (p 235 et suivantes), si certaines s'inscrivaient dans une perspective de réduction ou de questionnement de la réduction de la catégorisation scolaire. Parmi les 79 mesures préconisées, six visent à introduire plus d'informatique, de technologie ou de culture industrielle dans les filières de l'enseignement secondaire : cinq d'entre elles $\left(\mathrm{n}^{\circ} 17,22,23,24\right.$ et 25) s'intéressent à développer la transversalité de l'informatique et une seule $\left(\mathrm{n}^{\circ} 20\right)$ suggère la transversalité de l'ensemble des technologies (mais en fait sous la forme d'un court «module de culture industrielle »), mais rien n'est proposé sur la transversalité des « sciences » scolaires, à proprement parler.

On peut néanmoins noter trois autres recommandations qui semblent aller dans le sens de la diminution de la CSS par une ouverture générale aux sciences (et non simplement à l'informatique, la techno ou l'industrie), mais cependant de manière toujours ponctuelle : la $\mathrm{n}^{\circ} 15$ veut généraliser 1'EIST (enseignement intégré de sciences et de technologies) mais seulement jusqu'en classe de 3 eme, la $\mathrm{n}^{\circ} 28$ propose de « créer un concours pour promouvoir les projets scientifiques et techniques des jeunes dans toutes les classes du cours moyen de l'école primaire à la classe terminale (mais l'ambigüité subsiste pour savoir si cela concernerait vraiment toutes les filières ou seulement celles qui sont techniques et scientifiques, et si oui comment) enfin la no29 qui vise à systématiser, mais malheureusement à l’université et dans les écoles d'ingénieur, les enseignements interdisciplinaires car «il est indispensable de proposer une conception résolument transversales de ces formations qui n'impliquent pas uniquement sciences et technologie » (p130), sachant que là encore le texte est assez ambigu : le paragraphe correspondant laisse penser qu'il ne s'agit en fait que d'ouvrir à sens unique les filières sciences

\footnotetext{
${ }^{12}$ Celui-ci inclus «Les principaux éléments de mathématiques et la culture scientifique et technologique » et le fait de «Pratiquer une démarche scientifique et technologique, résoudre des problèmes » - la démarche scientifique est apparue dans les pratiques scolaires de façon institutionnelle dès les années 2002 (dans la suite de l'opération « Main à la Pâte » commencée en 1992) dans le primaire et dès 2006 dans le secondaire (socle commun de connaissances et de compétences, dont la compétence «Les principaux éléments de mathématiques et la culture

${ }^{13}$ Celle-ci est institutionnalisée à partir de septembre 2016 avec la réforme du collège mais avait eu ses prémisses en 2008 dans les programmes du collège avec les « thèmes de convergence ».
} 
et techno trop arides sans une ouverture sur cette dimension et non de faire cela symétriquement pour toutes les filières de SHS (puisque cette action est présentée comme une des actions de rénovation des enseignements de sciences et technologie).

\begin{tabular}{|c|c|}
\hline \multicolumn{2}{|l|}{ EXTRAITS DES RECOMMANDATIONS DU RAPPORT OPECST (2014) } \\
\hline I. Le développement du partage des cultures scientifique, technique et industrielle (CSTI) au sein du & système éducatif (pages 105 à 131) \\
\hline $\begin{array}{l}\text { 1) Inscrire la formation initiale et continue des enseignants dans une perspective de développement c } \\
\text { industrielle (cf. pages } 105 \text { à 110) }\end{array}$ & es cultures scientifique, technique et \\
\hline \multicolumn{2}{|c|}{ 2) Promouvoir une pédagogie au service des CSTI dans les différents niveaux d'enseignement (cf. pages 110 à 131) } \\
\hline \multicolumn{2}{|l|}{ - Dans les collèges (cf. pages 124 à 126) : } \\
\hline \multicolumn{2}{|l|}{ 15. Généraliser l'EIST (Enseignement intégré de science et de technologie) jusqu'en classe de troisième ; } \\
\hline \multicolumn{2}{|l|}{ - Dans les lycées (cf. pages 126 et 127): } \\
\hline \multicolumn{2}{|c|}{$\begin{array}{l}\text { 20. Étendre l'enseignement de la technologie à toutes les filières, conformément à l'avis de l'Académie des technologies du } 1^{\text {er }} \text { février } \\
2013 \text {; }\end{array}$} \\
\hline \multicolumn{2}{|c|}{$\begin{array}{l}\text { 22. Rendre obligatoire l'enseignement de l'informatique en seconde, première et en terminale sans exclure une option de spécialité plus } \\
\text { approfondie en terminale ; }\end{array}$} \\
\hline \multicolumn{2}{|c|}{ 23. Proposer un enseignement de l'informatique en première et en terminale ES ; } \\
\hline \multicolumn{2}{|l|}{ 24. Continuer et développer l'enseignement de l'informatique dans les séries techniques et professionnelles ; } \\
\hline \multicolumn{2}{|c|}{$\begin{array}{l}\text { 25. Étudier l'équilibrage horaire des disciplines requis par l'introduction de l'informatique avec, d'une part, un horaire spécifique } \\
\text { d'informatique et, d'autre part, la prise en compte de contenus informatiques au sein des autres disciplines et de leurs programmes. }\end{array}$} \\
\hline \multicolumn{2}{|c|}{$\begin{array}{l}\text { 28. Créer un concours pour promouvoir les projets scientifiques ou techniques des jeunes du cours moyen de l'école primaire à la classe } \\
\text { terminale, en s'inspirant de l'exemple allemand du Jugend forscht. }\end{array}$} \\
\hline - Dans les établissements d'enseignement supérieur (cf. pages 129 à 1 & \\
\hline \multicolumn{2}{|c|}{$\begin{array}{l}\text { 29. Systématiser l'organisation d'enseignements interdisciplinaires associant le plus souvent possible les différentes sciences dures et } \\
\text { biologiques, mais aussi les sciences humaines ; }\end{array}$} \\
\hline \multicolumn{2}{|c|}{ II. Réduire les inégalités d'accès au savoir scientifique et technologique (cf. pages 131 à 162) } \\
\hline \multicolumn{2}{|c|}{ 1) Corriger les effets pervers de la démocratisation ségrégative du système éducatif (cf. pages 131 à 147) } \\
\hline \multicolumn{2}{|c|}{ 2) Poursuivre résolument l'objectif d'égalité entre les femmes et les hommes (cf. pages 147 à 162) } \\
\hline \multicolumn{2}{|c|}{$\begin{array}{l}\text { III. Promouvoir une culture du dialogue apaisé entre les acteurs de la science, la technique, l'industrie et le public : une responsabilité } \\
\text { collective (cf. pages } 162 \text { à } 210 \text { ) }\end{array}$} \\
\hline \multicolumn{2}{|c|}{ 1) Développer les actions de médiation des chercheurs (cf. pages 162 à 177) } \\
\hline \multicolumn{2}{|c|}{ 2) Améliorer le traitement des CSTI par les médias (cf. pages 118 à 188) } \\
\hline \multicolumn{2}{|c|}{ 3) La contribution des entreprises au renforcement de la visibilité du volet industriel des CSTI (cf. pages 188 à 190) } \\
\hline \multicolumn{2}{|c|}{ 4) La place des citoyens dans le débat public (cf. page 190 à 201) } \\
\hline \multicolumn{2}{|c|}{ 5) Améliorer l'adéquation des décisions des autorités publiques aux exigences du débat public (cf. pages 201 à 210) } \\
\hline \multicolumn{2}{|c|}{ IV. Simplifier et améliorer la gouvernance (pages 211 à 232) } \\
\hline \multicolumn{2}{|l|}{ 1) La gouvernance nationale (page 211 à 225) } \\
\hline \multicolumn{2}{|l|}{ 2) La gouvernance territoriale (pages 225 à_232) } \\
\hline V. Modification de l'intitulé du rapport & \\
\hline
\end{tabular}

Tableau T2: Enoncés des huit recommandations (en caractères gras) parmi les 79 du rapport 2014 OPECST pouvant être vues en rapport avec la réduction de la CSS (sélection par l'auteur). Les titres de toutes les catégories et sous catégories du rapport ont été conservées (en caractères maigres). Source : http://www.senat.fr/rap/r13-274/r13-274.html

\subsection{La CSS, un enjeu aujourd'hui noyé dans d'autres priorités, sociales ou locales}

Cet exemple confirme que la fabrication par l'organisation des filières des lycées de la CSS n'est toujours pas identifiée comme un problème à combattre. Voilà donc qui renvoie à une question 
corollaire : Pourquoi la majorité des acteurs semblent encore aujourd'hui se satisfaire de la CSS et d'une CSTI qui ne la met pas en cause ? Nous proposons ici deux éléments de réponse à cette question, le premier concernant les stratégies développées par le ministère de l'éducation nationale, l'autre les acteurs de la CSTI.

1) L'éducation nationale donne depuis plus de trois décennies ${ }^{14}$ la priorité au traitement d'un autre problème : celui de la généralisation du baccalauréat et de l'accès à l'enseignement supérieur, qui se joue principalement par l'ouverture massive des bacs professionnels et technologiques. L'essentiel n'est donc pas la transformation des filières générales et la relation entre les bacs S, ES et L. On observe néanmoins des universités et écoles d'ingénieurs qui posent a posteriori cette question avec la création de filières «humanités et technologies » comme à l'université de technologie de Compiègne (UTC), ou des initiatives dans le monde de la recherche, comme celle de création de « centres de recherche interdisciplinaire », mais là encore c'est anecdotique en volume. On sent aussi qu'un frémissement pourrait venir des courants qui militent pour l'enseignement du codage informatique, mais force est de constater que toutes ces initiatives ne peuvent qu'être ponctuelles tant qu'il n'y aura pas de plan Marshall à échéance d'au moins une décennie, puisque les inerties des systèmes de formation des enseignants et de révision des cartes scolaires rendent impossible une évolution sans délai.

2) Les réseaux d'acteurs traditionnels de l'animation ou de l'action culturelle scientifique progressivement regroupées entre 1985 et 2005 derrière l'intitulé de CSTI se sont vus rejoints par une nouvelle génération encore plus disparate, composée pour beaucoup par les diplômés des récents Masters liés à la communication scientifique et technologique. Pour assurer leur avenir professionnel, bon nombre d'entre eux cherchent fort naturellement à faire exister et à pérenniser des créneaux solvables d'Outreach, de développement d'applications entre Serious Games et infos lettres ou des postures dites de curation de contenus gérées grâce à des agrégateurs. On entend ainsi beaucoup parler via des réseaux sociaux spécialisés d'une galaxie de manifestations accrochées sur des FabLabs au spectre de public particulièrement large entre stands de promotions d'applications smartphones et ateliers de bricolage se revendiquant d'une démarche de do it your self.

\footnotetext{
${ }^{14}$ Ce qui est une période de temps permettant une modulation des représentations de la population car elle recouvre plus d'une génération.
} 


\subsection{De nouveaux facteurs de complexification obscurcissent encore le paysage}

Face à cette situation, on peut légitiment se demander si la question de la CSS pourra être considérée comme un enjeu clef dans les années qui viennent. Rien n'est moins sûr, en particulier parce que la montée en puissance des interactions avec d'autres protagonistes plus spécialisés viennent encore complexifier encore le paysage.

On peut notamment citer parmi ceux-ci les acteurs militants de la promotion territoriales des innovations qui sont aujourd'hui poussés à se rassembler sous une nouvelle bannière associée par les financeurs publics nationaux et européens à la CSTI, à savoir celle dite de la « recherche et de l'innovation responsables» (RRI), dont l'intitulé semble avoir germé d'un transfert de la « responsabilité sociétale des entreprises » au monde de la recherche et développement. Ces acteurs sont bien sûr -au moins apparemment et localement- en résonnance avec de nombreux objectifs des CSTI de type 2, car l'encouragement des vocations n'est pas très loin de l'encouragement de l'innovation ou de type 4, car les démarches des Living Labs peuvent être vues comme proches d'une forme de transgression de la CSS.

Il en va de même avec le développement de services ou bureaux de CSTI au sein des établissements d'enseignement supérieur en résonnance avec des motivations liées à des stratégies de régionalisation de la formation, de fusion ou de recherches de financements argumentables en termes d'excellence (comme ceux du programme des investissements d'avenir), voire à des velléités d'être mieux appréciés dans des classements comme celui dit de Shanghaï. De fait, l'analyse de leurs discours et de leurs actions laisse entrevoir qu'ils se trouvent devoir osciller pragmatiquement entre des CSTI de type 2 (recherche d'étudiants pour leurs filières) et 3 (promotion, voire justification de leur pertinence vers les habitants de leurs territoires), complétés de type 5 (circulation des informations entre personnels des établissements. La complexification actuelle du paysage de la CSTI ne s'arrête pas là : les effets de la multiplication des prises de paroles sur la question du développement durable, liée bien sûr au phénomène du réchauffement climatique, mais aussi les effets récurrents des années thématiques témoignent sans ambiguïté de la sensibilité des acteurs aux effets d'aubaine, source indéniable d'amalgames er autres confusions. 


\section{Conclusion : construire un front uni contre la CSS}

On peut regarder cette évolution récente du paysage de la CSTI comme révélatrice du fait que malgré ses ambiguïtés ce champ apparait aujourd'hui paradoxalement comme un levier envié par de plus en plus d'acteurs diversifiés qui pensent y trouver un support propice à la transmission des valeurs qui leur sont importantes, comme par exemple celles favorables à l'esprit d'initiative et à l'innovation aussi bien que celles concernant le marketing technologique.

De fait, si l'on y prend garde, en raison de convergences purement tactiques de ces différents acteurs, les discours de CSTI vont continuer de se diversifier, risquant de réduire les possibilités de discours sur des enjeux communs au mieux à une simple rhétorique définissant la CSTI de manière auto-référente ou au pire à un conglomérat paradoxal, voire contradictoire. En conséquence, la complexité locale se cessera de s'accroitre, ce qui obscurcira de plus en plus toutes les velléités de lecture des enjeux macrosociaux.

Alors, plutôt que de laisser s'amalgamer sans précaution derrière la bannière de la CSTI de multiples acteurs prioritairement préoccupés par leurs propres enjeux locaux et disparates, ne faudrait-il pas mieux les convaincre tous -au fur et à mesure de leur ralliement à la sémantique de la CSTI- de faire aussi front autour d'une idée simple ? Celle que le caractère scientifique ne doit pas être vu comme une catégorie scolaire, mais comme une composante de toutes les qualifications professionnelles et une perspective appropriable par toutes et tous, comme un outil indispensable à l'esprit d'investigation et d'innovation dans la société de la connaissance. 


\section{Références bibliographiques}

ACKERMANN, W. \& DULONG, R. (1971). "Un nouveau domaine de recherche : la diffusion des connaissances scientifiques", in: Revue française de sociologie, 12-3, pp. 378-405, http://www.persee.fr/web/revues/home/prescript/article/rfsoc_0035-2969_1971_num_12_3_1996 [consulté le 27/03/2016].

BACHELARD, G. (1938). La formation de l'esprit scientifique, Paris : Vrin.

BANDURA, A. (2003). Auto-efficacité. Le sentiment d'efficacité personnelle. Bruxelles : de Boeck.

BARUK, S. (1973). Echec et maths. Seuil, Paris,

BRAND-GRAU A, CLAERR T \& SMITH P. (2015). «Sciences et techniques. Une culture à partager ». Culture et Recherche $\mathrm{n}^{\circ}$ 132, automne-hiver 2015-2016. Paris: Ministère de la culture et de la communication.

BENSAUDE-VINCENT, B. (2010). Splendeur et décadence de la vulgarisation scientifique. In dosssier «Les cultures des sciences en Europe » Questions de communication, $\mathrm{N}^{\circ} 17$.

BIGOT, R. \& al. (2013). La curiosité scientifique des français, Paris : CREDOC, Paris en ligne à http://www.credoc.fr/pdf/Rapp/R289.pdf [consulté le 27/03/2016].

BLANDIN, M.-C. \& RENAR, Y. (2003). La culture scientifique et technique, une priorité nationale, Paris : Sénat.

BROWN, P. (2010). Retour sur l'épidémiologie populaire, Traduction par François S et Akrich M. de Popular epidemiology revisited, 1997 Current Sociology 45 (3). In Akrich M, Barthe Y. et Remy, C. Sur la piste environnementale, menaces sanitaires et mobilisations profanes. Paris : Presses des Mines.

CALLON, M. (1998). Des différentes formes de démocratie technique, in Annales des Mines, 63-73

CONVERT, B. (2006). Les impasses de la démocratisation scolaire, sur une prétendue crise des vocations scientifiques. Paris : Éditions Raison d'agir.

DORAY, P., GEMME, B., GIBEAU, G. (2003). Culture scientifique et technique et navigation dans l'enseignement supérieur » in Schiele B., Jantzen, R. (dir.), Les Teritoires de la culture scientifique. Lyon : Presses universitaires de Lyon; Montréal : Les Presses de l'université de Montréal.

HCST \& CEREQ, (2013). Attractivité des carrières scientifiques et technologiques, Rapport de l'étude pilotée par le Haut Conseil de la Science et de la Technologie, HCST/CEREQ, Paris http://pmb.cereq.fr/doc_num.php?explnum_id=1236 [consulté le 27/03/2016].

EPSTEIN, S., (1996). Impure Science, AIDS, activism and the politics of Knowledge, Berkeley: University of California Press.

IGEN \& IGAEN, (2006). Evaluation des mesures prises pour revaloriser la série littéraire au lycée, Rapport 2006-044 IGEN, IGAEN : http://media.education.gouv.fr/file/63/8/3638.pdf 
p92-93 [consulté le 27/03/2016].

FELT, U. (2010). «Vers la construction d'un public européen ? Continuités et ruptures dans le discours politique sur les cultures scientifique et techniques ». In « Les cultures des sciences en Europe » Questions de communication, $\mathrm{N}^{\circ} 17$

JOUET, E. \& al. (2014). Nouvelles coopérations réflexives en santé, Paris : EAC.

KLINGER, C.-M. (2006). Challenging negative attitudes, low self-efficacy beliefs, and mathanxiety in pre-tertiary adult learners. Connecting voices: Practitioners, researchers \& learners in adult mathematics and numeracy, Melbourne: ALM Proceedings.

LABASSE, B. (1999). Observation sur la médiation des connaissances scientifiques et techniques, Rapport pour la DG XII de la Commission européenne. Publié en ligne http://spr.univ-lyon1.fr/GEV/Rapport.pdf [consulté le 27/03/2016].

LAS VERGNAS, O. (2006). Attractivité des études scientifiques : crise de foi, retour d'affection et main invisible du progrès : Communication à l'assemblée générale de l'association Planète sciences (mars 2004 FIAP-Paris) - Article réactualisé en février 2006. Assemblée générale de l'association Planète sciences mars 2004 : Et si le collège donnait le gout des sciences -FIAP, Mar 2004, Paris, France. 〈hal-00649467> [consulté le 27/03/2016].

LAS VERGNAS, O. (2011a). La culture scientifique et les non scientifiques, entre allégeance et transgression, Note d'HDR. Université de Paris Ouest-Nanterre-La Défense, Nanterre. En ligne à http://hdr.lasvergnas.eu [consulté le 27/03/2016].

LAS VERGNAS, O. (2011b). «L'institutionnalisation de la « culture scientifique et technique », un fait social français $(1970-2010)$. », Savoirs 3/2011 (n²7).

LAS VERGNAS, O. (2016). «De la médiation scientifique aux sciences dans la société, 30 ans d'ambiguïtés de l'action culturelle scientifique » in Caillet E. \& al. [dir]. La médiation culturelle : cinquième roue du carrosse ? Coll. Patrimoines et sociétés. Paris : L'Harmattan. p 177-187

LEVY-LEBLOND, J.-M. (2004). La science en mal de culture. Paris : Futuribles.

NIMIER, J. (1988). Les Modes de relations aux mathématiques. Paris : Méridiens Klincksieck,

OPECST, sous la direction de Olivier, M. et Leleux, J-P. (2014). Faire connaitre et partager les cultures scientifiques techniques et industrielles, un impératif. Paris : Les rapports de l'OPECST $315+85$ pages

PRICE, D. DE SOLLA, (1963). Little Science, Big Science. New-York and London: Columbia University Press.

RERS (2014). Ministère de l'éducation Nationale : repères et résultats statistiques, Paris : MEN

SCHIELE, B. (2005). "Publiciser la science, pourquoi faire ? Revisiter la notion de culture scientifique et technique", in Pailliart I. (dir.), La publicisation de la science, Grenoble : PUG, p. $11-51$. 
SNOW, C. (1968)., Les deux cultures [traduction de The two cultures, parution originale 1959, CUP]. Paris : Pauvert.

SUINN, R.-M. \& WINSTON, E.-H. (2003). The mathematics anxiety rating scale, a brief version: Psychol.Reports: vol 92.

TEMKENG A. (en cours), Déterminants motivationnels et apprenance vis-à-vis des savoirs scientifiques scolaires au Cameroun (thèse, ED139, sous la Dir de Las Vergnas O.). Nanterre : CREF-AFA-UPX. 